\title{
Aspectos toxicológicos e ocorrência dos bifenilos policlorados em alimentos
}

\author{
Toxicological aspects and occurrence of polychlorinated biphenyls in food
}

\author{
Susana MohrI Ijoni Hilda CostabeberII
}

\section{- REVISÃO BIBLIOGRÁFICA -}

RESUMO

Os PCBs são compostos orgânicos sintéticos que foram muito utilizados na indústria como fluídos dielétricos em transformadores e capacitores, tintas e óleos lubrificantes hidráulicos. Após serem detectados no meio ambiente no final dos anos 60, em seguida foram identificados como contaminantes de todo o ecossistema global, incluindo o ar, água, solo, plantas, animais, seres humanos e alimentos, o que levou à sua proibição em 1977, nos Estados Unidos, e, em 1981, no Brasil. Porém, sua utilização ainda é permitida no nosso país em equipamentos eletrônicos antigos, até que seja realizada a sua substituição por produto isento de PCBs. Esse fato tem resultado em diversos episódios de utilização, armazenamento e descarte inadequados, contaminando o meio ambiente, os animais, os seres humanos e toda a cadeia alimentar. Estudos demonstram que esses compostos são capazes de causar relevantes alterações, tais como neurotoxicidade, disrupção endócrina, imunossupressão, entre outros. Segundo a IARC, os PCBs estão classificados no Grupo $2 A$ (provavelmente carcinogênicos para seres humanos), com exceção do PCB 126, que pertence ao Grupo 1 (carcinogênico para seres humanos). Como estes compostos são altamente lipofílicos, acumulam-se na cadeia alimentar e têm sido detectados nos mais diversos tipos de alimentos com bastante frequência, sendo esta a principal fonte de contaminação dos seres humanos. Esta revisão bibliográfica tem por objetivo apresentar os principais aspectos relacionados aos bifenilos policlorados em alimentos, incluindo suas características, aspectos toxicológicos e ocorrência.

Palavras-chave: bifenilos policlorados, poluentes orgânicos persistentes, contaminação de alimentos, segurança alimentar, toxicidade.

\begin{abstract}
PCBs are synthetic organic compounds that were widely used in industry as dielectric fluids in transformers and capacitors, paints and lubricants hydraulic oils. After being detected in the environment on the end of 60, then they were identified as contaminants in the whole ecosystem, including the air, water, soil, plants, animals, humans and food, which led to its prohibition in 1977, in the United States, and in 1981, in Brasil. Nevertheless, its use is still allowed in our country in old electronics, until its replacement by other free PCB product. This has resulted in several use episodes, inadequate storage and disposal, contaminating the environment, animals, humans and all the food chain. Studies show that these compounds are able to cause significant changes, such as neurotoxicity, endocrine disruption, and immunosuppression, among others. According to the IARC, PCBs are classified in Group 2A (probably carcinogenic to humans), with the exception of PCB 126, which belongs to Group 1 (carcinogenic to humans). Because their high lipophilicity, accumulates in the food chain and, consequently, has been detected often in many kinds of food, which is the main source of human contamination. The aim of this review is to present the mains aspects related to polychlorinated biphenyls in foods, including their characteristics, toxicological aspects and occurrence.
\end{abstract}

Key words: polychlorinated biphenyls, persistent organic pollutants, food contamination, food security, toxicity.

\section{INTRODUÇÃO}

A busca por alimentos seguros pode ser considerada na atualidade como uma forma de

'Departamento de Tecnologia e Ciência dos Alimentos, Universidade Federal de Santa Maria (UFSM), Santa Maria, RS, Brasil. "Departamento de Morfologia, UFSM, Avenida Roraima, 1000, Prédio 19, 97105-900, Santa Maria, RS, Brasil. E-mail: ijonicostabeber@gmail.com. Autor para correspondência. 
conhecimento aplicado à qualidade de vida. Na visão atual do consumidor, o conceito de qualidade de um alimento não engloba somente as características de sabor, aroma e aparência, mas também a preocupação de que não cause danos à saúde e seja livre de contaminantes físicos, químicos e microbiológicos. Disponibilizar alimentos seguros para a população é uma tarefa que envolve todas as esferas do governo, bem como as empresas e estabelecimentos privados afins ao processo.

A presença de contaminantes químicos nos alimentos pode causar vários danos, incluindo reações de hipersensibilidade e toxicidade, que podem ocorrer de forma aguda, crônica e/ou retardada, como a ação carcinogênica (MIDIO \& MARTINS, 2000). Dentre esses contaminantes químicos, destacam-se os bifenilos policlorados (PCBs), compostos pertencentes à classe dos poluentes orgânicos persistentes (POPs), presentes em inúmeras amostras indicadoras da contaminação ambiental. Sua descoberta ocorreu há mais de cem anos, tendo sido amplamente utilizados pela indústria durante décadas em diversas aplicações (ERICKSON \& KALEY II, 2011). Inicialmente detectados no meio ambiente no final dos anos 60, foram identificados como contaminantes em quase todos os componentes do ecossistema global, resultando em inúmeros efeitos tóxicos e biológicos. Estes compostos são comprovadamente causadores de problemas reprodutivos, imunológicos e de danos ao fígado (SAFE et al., 1985). As proporções da dispersão e transferência desses compostos para os animais, vegetais e seres humanos são difíceis de serem estimadas (WHO, 1993).

O objetivo desta revisão bibliográfica é apresentar os principais aspectos relacionados aos bifenilos policlorados em alimentos, incluindo suas características, aspectos toxicológicos e ocorrência.

Características gerais dos bifenilos policlorados

Os PCBs são hidrocarbonetos clorados, fabricados comercialmente pela progressiva cloração do bifenil na presença de um catalisador. Dependendo das condições de reação, as proporções de cloração podem variar entre 21 e 68\%. Quimicamente, são caracterizados por dois anéis benzênicos unidos por uma ligação C-C, tendo a fórmula geral de $\mathrm{C}_{12} \mathrm{H}_{(10-\mathrm{n})} \mathrm{Cl}_{\mathrm{n}}$ $(1=n=10)$. Suas moléculas podem apresentar diversas substituições em relação à quantidade de átomos de cloro, variando de 1 a 10 átomos, podendo ser obtidas até 209 estruturas diferentes, denominadas de congêneres (PEREIRA, 2004; ERICKSON \& KALEY II, 2011). Os anéis de benzeno podem girar em torno da ligação que os une, resultando em duas conformações espaciais: planar ou coplanar. A conformação planar apresenta átomos de cloro na posição orto (2,2'), enquanto que a coplanar não possui átomos de cloro nessa posição (PENTEADO \& VAZ, 2001). A estrutura dos PCBs possui uma considerável resistência a ácidos, bases, altas temperaturas e correntes elétricas, além de ser uma substância não inflamável (OMAYE, 2004). São praticamente insolúveis em água e facilmente solúveis em carboidratos, gorduras e outros compostos orgânicos, sendo rapidamente absorvidos por tecidos gordurosos (WHO, 1993; SILVA et al., 2007).

Devido ao grande emprego de PCBs, a produção mundial acumulada foi de aproximadamente 1.200.000 toneladas. Destes, cerca de $60 \%$ foram utilizados em transformadores e capacitores, $15 \%$ como fluídos de transferência de calor e $25 \%$ como aditivos na formulação de plastificantes, tintas, adesivos e pesticidas (PENTEADO \& VAZ, 2001). Segundo OMAYE (2004), a produção de PCBs nos Estados Unidos foi interrompida em 1977, entretanto, mais de 500.000 toneladas foram produzidas naquele país. Estima-se que dois terços foram degradados ou destruídos, mas, devido a seu alto grau de estabilidade, cerca de 200.000 toneladas ainda podem estar presentes no meio ambiente.

No Brasil, não se tem registros de sua produção, sendo o produto importado principalmente dos Estados Unidos e Alemanha. A Portaria Interministerial 19, de 29 de janeiro de 1981, estabeleceu a proibição da fabricação, comercialização e uso de PCBs em todo território nacional, porém permite que os equipamentos já instalados continuem em funcionamento até a sua substituição integral ou troca do fluído dielétrico por produto isento de PCBs (BRASIL, 1981).

A contaminação ambiental ocorre principalmente a partir do descarte de equipamentos elétricos antigos em locais não apropriados, contaminando o solo e as águas. Também ocorre durante a incineração do lixo industrial e municipal, pois os incineradores não são efetivos em sua destruição. Os PCBs são inflamáveis a altas temperaturas e os produtos de sua combustão podem ser muito mais perigosos do que o produto original, como os furanos (PCDFs). A combustão dos produtos de grau técnico contendo PCBs e benzenos clorados, como os fluídos dielétricos, pode também produzir as dioxinas (PCDDs) (WHO, 2003; PEREIRA, 2004). Segundo a legislação do Conselho Nacional do Meio Ambiente (BRASIL, 2002), todo sistema de tratamento térmico para resíduos industriais deverá atingir a taxa de eficiência de destruição e remoção (EDR) superior ou igual a 99,99\% para os PCBs, com as câmaras 
operando a uma temperatura mínima de $800^{\circ} \mathrm{C}$. Por esse motivo, muitos equipamentos desativados contendo PCBs ainda continuam armazenados em depósitos, como ocorreu recentemente no Porto de Rio Grande (RS), o qual foi autuado e multado pelo IBAMA por manter 21 toneladas de óleo Ascarel (denominação comercial do óleo de PCBs no Brasil) armazenadas em transformadores desativados e tonéis. Isso demonstra que, apesar de esse produto estar proibido há quase três décadas no país, o risco de contaminação ambiental ainda é muito grande.

Aspectos toxicológicos dos bifenilos policlorados O homem pode contaminar-se com PCBs a partir do meio ambiente, por ingestão através de alimentos, inalação, absorção dérmica e processos de combustão inadequados. As vias de entrada dos PCBs no organismo humano são através do trato gastrointestinal, pulmões ou epiderme (WHO, 1993). Segundo ROSS (2004), a exposição humana aos PCBs ocorre principalmente pela ingestão acidental de alimentos com baixos níveis de contaminação. $\mathrm{O}$ mecanismo e a cinética da biotransformação dos PCBs dependem de inúmeros fatores, incluindo a capacidade metabólica do organismo e a estrutura do congênere de PCB (MAERVOET et al., 2004). Aqueles congêneres que não contêm átomos de cloro na posição orto, mas possuem átomos de cloro na posição para e têm no mínimo um par de cloros na posição meta, são considerados os mais tóxicos, devido à alta afinidade com o receptor Ah (aryl hydrocarbon), tais como o 3,3',4,4' -tetraclorobifenil (PCB 77), 3,4,4',5tetraclorobifenil (PCB 81), 3,3',4,4',5-pentaclorobifenil (PCB 126) e 3,3',4,4',5,5'-hexaclorobifenil (PCB 169). Os mecanismos de ação bioquímico e toxicológico desses compostos são semelhantes aos da 2,3,7,8 tetraclorodibenzeno-p-dioxina (TCDD) (SAFE et al., 1985; SILVA et al., 2007) e, por esse motivo, estes congêneres são chamados de $\mathrm{PCBs}$ semelhantes às dioxinas (DL-PCBs: dioxin-like PCBs). Já as dioxinas são formadas indesejavelmente a partir dos PCBs, sendo que a maioria dos casos de contaminação resulta de sua formação a partir de processos industriais, incineração do lixo industrial (WEBER et al., 2008), ou incêndio acidental (VASSILIADOU et al., 2009). O termo "dioxinas" compreende um total de 210 congêneres, os quais estão quimicamente e estruturalmente relacionados aos hidrocarbonetos aromáticos clorados, incluindo 75 congêneres de PCDD e 135 congêneres de PCDF. Seu potencial toxicológico difere significativamente, dependendo do grau de cloração e da posição dos átomos de cloro em seus dois anéis aromáticos. Todos os 17 congêneres de PCDD/PCDFs com substituições nas posições $2,3,7$ e 8 possuem um perfil toxicológico semelhante, os quais incluem toxicidade dérmica, carcinogenicidade, imunotoxicidade e toxicidade no sistema reprodutivo (SAFE, 1986). Porém, os congêneres 2,3,7,8-tetraclorodibenzeno- $p$ dioxina, 2,3,4,7,8-pentaclorodibenzenofurano, bem como o PCB 126 (3,3',4,4',5-pentaclorobifenil) foram recentemente classificados pela International Agency for Research on Cancer (IARC) no Grupo 1 (comprovadamente carcinogênicos para os seres humanos) (BAAN et al., 2009).

A maioria das misturas comerciais de $\mathrm{PCBs}$, porém, está baseada nos sete indicadores $(28,52,101$, $118,138,153$ e 180). O somatório de seis desses indicadores, com exceção do PCB 118, representa cerca de $50 \%$ da contaminação dos alimentos por PCBs não semelhantes às dioxinas (NDL-PCBs: non-dioxin like PCBs) (EFSA, 2005; FERRANTE et al., 2011). Os NDLPCBs são reconhecidamente menos tóxicos que os congêneres DL, embora sejam igualmente perigosos devido ao fato de serem comumente encontrados nos seres humanos, animais selvagens e peixes (FERRANTE et al., 2010), expostos através da cadeia alimentar em concentrações muito maiores do que os DL-PCBs (STORELLI \& PERRONE, 2010). Está cada vez mais claro que a maioria dos PCBs que possuem átomos de cloro na posição orto (NDL-PCBs), os quais possuem pequena afinidade pelos receptores $\mathrm{Ah}$ e que se pensava ser biologicamente inativos, estão envolvidos em relevantes alterações, tais como neurotoxicidade, disrupção endócrina e imunossupressão (SELGRADE, 2007). A respeito dos efeitos dos NDL-PCBs no sistema imune, alguns estudos in vivo demonstraram supressão na produção de anticorpos e da resposta imune durante a fagocitose, além da diminuição na resistência a infecções em seres humanos e animais expostos (LUEBKE et al., 2004; LEVIN etal., 2005; DALLAIRE etal., 2006; SELGRADE, 2007). As condições que levam à liberação e redistribuição desses compostos podem fornecer informações sobre seu perfil toxicológico, cinética e efeitos patológicos em seres humanos (KIM et al., 2011). Uma perda de peso moderada foi associada com o aumento na concentração de PCBs no plasma sanguíneo, sugerindo que a redução desses poluentes na massa de gordura corporal pode estar associada ao aumento da lipólise (HUE et al., 2006).

Os compostos mais clorados são mais persistentes, sendo absorvidos pelo sistema intestinal, acumulando-se principalmente nos tecidos gordurosos e fígado, nos quais podem permanecer por diversos anos. Já as formas menos cloradas entram na circulação hepática portal, são submetidas a um metabolismo 
primário e podem ser metabolizadas e excretadas (ATSDR, 2000). As principais rotas de eliminação dos PCBs são as fezes, a urina e o leite materno (WHO, 2003).

Ao nível de exposição atual, é pouco provável que os PCBs causem graves efeitos a curto prazo na saúde humana. Alguns estudos demonstram que a exposição direta pode causar problemas na pele, irritação no nariz e pulmões, mal estar gastrointestinal, alterações sanguíneas e hepáticas, além de depressão e fadiga, principalmente em locais onde são mantidos ou consertados transformadores que ainda contém PCBs (ATSDR, 2000). Já os efeitos na saúde a longo prazo, porém, são inúmeros. Segundo a International Agency for Research on Cancer (IARC), os PCBs estão classificados no Grupo 2A (provavelmente, carcinogênicos para seres humanos), com exceção do PCB 126, já mencionado anteriormente (Grupo 1). Além disso, diversos estudos têm sugerido que a exposição aos PCBs está associada ao aumento do risco do câncer de mama (RECIO-VEGA et al., 2011), câncer do trato biliar (ADENUGBA et al., 2009), câncer de fígado, estômago, intestino e próstata (PRINCE et al., 2006), entre outros.

Ocorrência dos bifenilos policlorados em alimentos

A entrada dos PCBs na cadeia alimentar deve-se principalmente as suas propriedades físicoquímicas. Esses compostos podem sofrer um processo de bioconcentração, no qual ocorre um acúmulo resultante da absorção e eliminação simultâneas, ou uma biomagnificação, resultado do processo de acúmulo da concentração de PCBs nos tecidos dos organismos vivos na passagem de cada nível trófico da cadeia alimentar (PENTEADO \& VAZ, 2001). Devido ao fato de que a mais importante via de exposição humana aos PCBs é a ingestão de alimentos contaminados, muitos estudos têm sido realizados para detectar os níveis de contaminação dos alimentos. $\mathrm{O}$ maior número de pesquisas concentra-se em países fabricantes de PCBs, como Itália, Alemanha, Japão, Estados Unidos, além de locais com ocorrência de acidentes ambientais com esses materiais.

É sempre preferível que o perigo químico não esteja presente no alimento. Porém, como nem sempre isso é possível, os órgãos governamentais estabelecem os níveis que são aceitos, ou seja, a concentração do perigo que é considerada "aceitável", como limite de referência. A legislação brasileira prevê limites de referência para PCBs em carne $\left(200 \mu \mathrm{g} \mathrm{kg}^{-1}\right.$ de gordura), leite $\left(100 \mu \mathrm{g} \mathrm{L}^{-1}\right)$ e peixe de cultivo $\left(15 \mu \mathrm{g} \mathrm{kg}^{-1}\right)$ (BRASIL, 2011). Já os limites máximos aceitos pela União Europeia são expressos em TEQ (total de toxicidade equivalente) para o somatório de PCDDs e PCDFs e para o somatório de PCDDs e DL-PCBs (CEE, 2006).
A tabela 1 apresenta a ocorrência de bifenilos policlorados em alimentos de diversos países, com estudos realizados entre os anos de 2006 e 2011. Com relação à carne, produtos cárneos e fígado, observa-se que a salsicha hot-dog analisada no Brasil foi a que apresentou a maior contaminação (COSTABEBER et al., 2006). Já o fígado bovino, que é um dos locais em que os PCBs armazenam-se no organismo, apresentou baixa contaminação quando relacionado aos outros alimentos do grupo (TURRIO-BALDASSARI, 2009). No grupo dos pescados, uma concentração considerável foi detectada no salmão proveniente da Bélgica (VOORSPOELS et al., 2008), seguido do salmão da Dinamarca (FROMBERG et al., 2011). Esse mesmo estudo na Bélgica também detectou uma alta contaminação em ovos. Já em outro estudo com ovos, realizado mais recentemente na Dinamarca, a contaminação foi menor (FROMBERG et al., 2011). Em relação aos lácteos, uma grande contaminação foi detectada no leite bovino do Irã (BAYAT et al., 2011), seguido pelo leite do Brasil (HECK et al., 2007). Já os leites provenientes da Dinamarca (FROMBERG et al., 2011) e da Itália (TURRIOBALDASSARI et al., 2009) apresentaram contaminações inferiores. No que diz respeito aos queijos, os provenientes do Brasil (SANTOS et al., 2006) apresentaram contaminação superior quando comparados aos provenientes da Dinamarca (FROMBERG et al., 2011). O leite materno, uma das principais vias de eliminação dos PCBs do organismo, apresentou grande quantidade de resíduos nos estudos realizados recentemente na Espanha (GÓMARA et al., 2011) e no Japão (TOKADA et al., 2010). Porém, comparando-se com o estudo realizado na China, o valor apresenta-se inferior (XING et al., 2009). Suplementos alimentares a base de óleo de fígado de bacalhau e azeite de oliva virgem também foram analisados na Espanha, constatando-se uma alta concentração de PCBs (YAGÜE et al., 2005; MARTÍ et al., 2010).

Com base nesses dados, pode-se analisar melhor a situação da contaminação dos alimentos do Brasil. Embora ainda haja poucos estudos sobre o tema, pode-se observar que os alimentos analisados apresentaram uma contaminação significativa (leite bovino, queijo e salsicha hot-dog), uma vez que não existiram fábricas de PCBs no país. O descarte inadequado de resíduos e equipamentos elétricos antigos talvez possa ser a maior causa dessa contaminação. Um estudo recente realizado no estado do Rio Grande do Sul demonstrou que o índice de contaminação por PCBs em soro de cordão umbilical de recém nascidos foi considerado elevado, quando comparado com estudos da Europa e Estados Unidos (MOHR et al., 2011), indicando que essa contaminação também está presente nos seres humanos. 
Tabela 1 - Ocorrência de bifenilos policlorados em alimentos de diversos países.

\begin{tabular}{|c|c|c|c|c|}
\hline País & Alimento & Congêneres de PCBs analisados & $\sum$ PCBs & Referência \\
\hline Japão & Peito de frango & $\begin{array}{l}36 \text { congêneres: } 18,37,44,49,52,70,74,77,81,87,99 \text {, } \\
101,105,114,118,119,123,126,128 / 158,138,151,156, \\
157 / 153,167,168,169,170,177,180,183,187,189,194, \\
199\end{array}$ & $3,06 \mathrm{ng} \mathrm{g}^{-1}$ & QIN et al. (2011) \\
\hline Brasil & Salsicha hot-dog & 6 congêneres: $10,28,52,138,153,180$ & $\begin{array}{l}31,19 \mathrm{ng} \mathrm{g}^{-1} \\
\text { gordura }\end{array}$ & $\begin{array}{l}\text { COSTABEBER et al. } \\
\text { (2006) }\end{array}$ \\
\hline Brasil & Carne bovina & 6 congêneres: $10,28,52,138,153,180$ & $\begin{array}{l}2,82 \mathrm{ng} \mathrm{g}^{-1} \\
\text { gordura }\end{array}$ & $\begin{array}{l}\text { COSTABEBER et al. } \\
\text { (2006) }\end{array}$ \\
\hline Itália & Fígado bovino & 6 congêneres: $28,52,101,138,153,180$ & $\begin{array}{l}0,85 \mathrm{a}^{-1} \\
1,82 \mathrm{ng} \mathrm{g}^{-1} \\
\text { gordura }\end{array}$ & $\begin{array}{l}\text { TURRIO-BALDAS- } \\
\text { SARI et al. (2009) }\end{array}$ \\
\hline Argentina & Peixe (truta) & 9 congêneres: $44,87,110,118,138,149,153,180,187$ & $\begin{array}{l}4,7 \mathrm{ng} \mathrm{g}^{-1} \\
\text { gordura }\end{array}$ & $\begin{array}{l}\text { ONDARZA et al. } \\
\qquad(2011)\end{array}$ \\
\hline Bélgica & Salmão & $\begin{array}{l}23 \text { congêneres: } 28,52,74,95,99,101,105,110,118,128 \text {, } \\
138 / 163,149,153,156,170,180,183,187,194,196 / 203 \text {, } \\
199\end{array}$ & $24 n g g^{-1}$ & $\begin{array}{l}\text { VOORSPOELS et al. } \\
\qquad(2008)\end{array}$ \\
\hline Dinamarca & Salmão & $\begin{array}{l}10 \text { congêneres: } 28,52,101,105,118,138,153,156,170 \text {, } \\
180\end{array}$ & $15,8 \mathrm{ng} \mathrm{g}^{-1}$ & $\begin{array}{l}\text { FROMBERG et al. } \\
\qquad(2011)\end{array}$ \\
\hline Bélgica & Ovos & $\begin{array}{l}23 \text { congêneres: } 28,52,74,95,99,101,105,110,118,128 \text {, } \\
138 / 163,149,153,156,170,180,183,187,194,196 / 203 \text {, } \\
199\end{array}$ & $35 \mathrm{ng} \mathrm{g}^{-1}$ & $\begin{array}{l}\text { VOORSPOELS et al. } \\
\qquad(2008)\end{array}$ \\
\hline Dinamarca & Ovos & $\begin{array}{l}10 \text { congêneres: } 28,52,101,105,118,138,153,156,170 \text {, } \\
180\end{array}$ & $4,7 \mathrm{ng} \mathrm{g}^{-1}$ & $\begin{array}{l}\text { FROMBERG et al. } \\
\qquad(2011)\end{array}$ \\
\hline Irã & Leite bovino & 6 congêneres: $28,52,101,138,153,180$ & $\begin{array}{l}27,06 \mathrm{ng} \mathrm{g}^{-1} \\
\text { gordura }\end{array}$ & BAYAT et al. (2011) \\
\hline Brasil & Leite bovino & 5 congêneres: $10,28,52,138,180$ & $\begin{array}{l}15,5 \mathrm{ng} \mathrm{g}^{-1} \\
\text { gordura }\end{array}$ & HECK et al. (2007) \\
\hline Dinamarca & Leite bovino & $\begin{array}{l}10 \text { congêneres: } 28,52,101,105,118,138,153,156,170 \text {, } \\
180\end{array}$ & $\begin{array}{l}4,4 \mathrm{ng} \mathrm{g}^{-1} \\
\text { gordura }\end{array}$ & $\begin{array}{l}\text { FROMBERG et al. } \\
\qquad(2011)\end{array}$ \\
\hline Itália & Leite bovino & 6 congêneres: $28,52,101,138,153,180$ & $\begin{array}{l}0,18 \text { a } \\
0,48 \text { ng g }^{-1} \\
\text { gordura }\end{array}$ & $\begin{array}{l}\text { TURRIO-BALDAS- } \\
\text { SARI et al. (2009) }\end{array}$ \\
\hline Brasil & Queijo & 6 congêneres: $10,28,52,138,153,180$ & $\begin{array}{l}30,84 \mathrm{ng} \mathrm{g}^{-1} \\
\text { gordura }\end{array}$ & SANTOS et al. (2006) \\
\hline Dinamarca & Queijo & $\begin{array}{l}10 \text { congêneres: } 28,52,101,105,118,138,153,156,170 \text {, } \\
180\end{array}$ & $\begin{array}{l}6,8 \mathrm{ng} \mathrm{g}^{-1} \\
\text { gordura }\end{array}$ & $\begin{array}{l}\text { FROMBERG et al. } \\
\qquad(2011)\end{array}$ \\
\hline Espanha & Leite materno & $\begin{array}{l}24 \text { congêneres: } 28,52,77,81,95,101,105,114,118,123 \text {, } \\
126,132,138,149,153,156,157,167,169,170,180,183 \text {, } \\
189,194\end{array}$ & $\begin{array}{l}125 \mathrm{ng} \mathrm{g}^{-1} \\
\text { gordura }\end{array}$ & $\begin{array}{l}\text { GÓMARA et al. } \\
\qquad(2011)\end{array}$ \\
\hline Japão & Leite materno & 6 congêneres: $28,52,101,138,153,180$ & $\begin{array}{l}48,28 \mathrm{ng} \mathrm{g}^{-1} \\
\text { gordura }\end{array}$ & $\begin{array}{l}\text { TOKADA et al. } \\
\qquad(2010)\end{array}$ \\
\hline China & Leite materno & $\begin{array}{l}36 \text { congêneres: } 18,37,44,49,52,70,119,123,126, \\
128 / 158,138,151,156,74,77,81,87,99,101,105,114, \\
118,157 / 153,167,168,169,170,177,180,183,187,189, \\
194,199\end{array}$ & $\begin{array}{l}9,50 \mathrm{ng} \mathrm{g}^{-1} \\
\text { gordura }\end{array}$ & XING et al. (2009) \\
\hline Japão & Amêndoa & $\begin{array}{l}36 \text { congêneres: } 18,37,44,49,52,70,74,77,81,87,99 \text {, } \\
101,105,114,118,119,123,126,128 / 158,138,151,156, \\
157 / 153,167,168,169,170,177,180,183,187,189,194, \\
199\end{array}$ & $2,69 \mathrm{ng} \mathrm{g}^{-1}$ & QIN et al. (2011) \\
\hline Espanha & $\begin{array}{l}\text { Azeite de oliva } \\
\text { (virgem) }\end{array}$ & 5 congêneres: $28,52,138,153,180$ & $37,1 \mathrm{ng} \mathrm{g}^{-1}$ & YAGÜE et al. (2005) \\
\hline Espanha & $\begin{array}{l}\text { Supl.alimentar } \\
\text { (óleo bacalhau) }\end{array}$ & 7 congêneres: $28,52,101,118,138,153,180$ & $\begin{array}{l}49,7 \mathrm{a} \\
98,3 \mathrm{ng} \mathrm{g}^{-1}\end{array}$ & MARTÍ et al. (2010) \\
\hline
\end{tabular}




\section{CONCLUSÃO}

Os aspectos toxicológicos dos PCBs são bastante variáveis, devido à quantidade e posição dos átomos de cloro em sua molécula. Os DL-PCBs são os mais tóxicos, pois possuem alta afinidade com os receptores $\mathrm{Ah}$, além de apresentarem um mecanismo de ação toxicológico semelhante à dioxina 2,3,7,8TCDD. Já os congêneres NDL-PCBs, apesar de apresentarem uma toxicidade menor, são igualmente perigosos, pois foram os mais utilizados em misturas comerciais e, consequentemente, os mais detectados em amostras ambientais, seres humanos, animais e alimentos. Estudos já comprovaram que esses congêneres, apesar de possuírem pequena afinidade pelos receptores Ah, estão envolvidos em mecanismos de neurotoxicidade, disrupção endócrina e imunossupressão. Apesar de os PCBs terem sido proibidos há mais de três décadas, seus resíduos ainda são detectados nos mais diversos tipos de alimentos de vários países, inclusive do Brasil. A maior causa talvez esteja nos frequentes acidentes de armazenamento e descarte incorretos, somado a sua grande persistência e bioacumulação. Sua ocorrência em alimentos do mundo inteiro demonstra que o problema é global, incluindo países desenvolvidos e em desenvolvimento. Na maioria das referências consultadas, não se observou uma diminuição nos níveis de PCBs com o passar dos anos, como era de se esperar. As proporções do efeito da contaminação dos PCBs nos seres humanos ainda são difíceis de ser estimadas, porém estudos já comprovaram o seu poder carcinogênico, mutagênico e teratogênico. A necessidade da realização de mais pesquisas no país é evidente, porém os custos para este tipo de análise ainda são muito altos, o que justifica os poucos trabalhos dentre as referências consultadas. Contudo, mais pesquisas sobre a ocorrência dos PCBs em alimentos fazem-se necessárias no mundo inteiro, devido à grande variabilidade nos resultados obtidos dentro da literatura consultada.

\section{AGRADECIMENTOS}

Os autores agradecem ao Conselho Nacional de Desenvolvimento Científico e Tecnológico (CNPq - Processo 578343/2008-8). S. Mohr é bolsista da Coordenação de Aperfeiçoamento de Pessoal de Nível Superior (CAPES). I. Costabeber é bolsista de Produtividade em Pesquisa (CNPq).

\section{REFERÊNCIAS}

ADENUGBA, A. et al. Polychlorinated biphenyls in bile of patients with biliary tract cancer. Chemosphere, v.76, n.6, p.841-846, 2009. Disponível em: <http://www.sciencedirect.com/
science/article/pii/S004565350900441X>. Acesso em: 28 nov. 2011. doi: 10.1016/j.chemosphere.2009.04.003.

ATSDR. Agency for Toxic Substances and Disease Registry. Toxicological profile for polychlorinated biphenyls (PCBs). Atlanta, GA: U.S. Department of Health and Human Services, Public Health Service, 2000. Acesso em: 28 nov. 2011. Online. Disponível em: <http://www.atsdr.cdc.gov/ toxprofiles/tp.asp?id=142\&tid=26>.

BAAN, R. et al. A review of human carcinogens - Part F: chemical agents and related occupations. Lancet Oncology, v.10, n.12, p.1143-1144, 2009. Disponível em: <http:// www.thelancet.com/journals/lanonc/article/PIIS 1470 2045(09)70358-4/fulltext>. Acesso em: 28 nov. 2011. doi: 10.1016/S1470-2045(09)70358-4.

BAYAT, S. et al. Survey of organochlorine pesticides and polychlorinated biphenyls in commercial pasteurized milk in Iran. Environmental Monitoring and Assessment, v.175, p.469-474, 2011. Disponível em: <http://www.springerlink.com/ content/e67811h228744614/>. Acesso em: 28 nov. 2011. doi: 10.1007/s10661-010-1544-y.

BRASIL. Portaria Interministerial n.19 de 29 de janeiro de 1981. Acesso em: 28 nov. 2011. Online. Disponível em: $<\mathrm{http}: / / \mathrm{ww}$.ipef.br/legis lacao/bdlegis lacao/ detalhes.asp? Id=235>.

BRASIL. Ministério do Meio Ambiente. Resolução n.316 de 29 de outubro de 2002. Diário Oficial da República Federativa do Brasil, Poder Executivo, Brasília, DF, 20 de novembro de 2002, seção 1 .

BRASIL. Ministério da Agricultura, Pecuária e Abastecimento. Programa de controle de resíduos de contaminantes em carnes, leite, mel, ovos e pescado - PNCRC/2011. Instrução Normativa n.24 de 9 de agosto de 2011.

CEE. Comissão das Comunidades Européias. Regulamento (CE) n.1881/ 2006 de 19 de dezembro de 2006. Fixa os teores máximos de certos contaminantes presentes em gêneros alimentícios. Acesso em: 28 nov. 2011. Online. Disponível em: $<$ http://eur-lex.europa.eu/ LexUriServ/LexUriServ.do?uri=OJ:L:2006:364:0005:0024:PL:PDF>.

COSTABEBER, I. et al. Levels of polychlorinated biphenyls (PCBs) in meat and meat products from the state of Rio Grande do Sul, Brazil. Food and Chemical Toxicology, v.44, n.1, p.1-7, 2006. Disponível em: <http://www.sciencedirect.com/ science/article/pii/S0278691505000207>. Acesso em: 28 nov. 2011. doi: 10.1016/j.fet.2005.01.005.

DALLAIRE, F. et al. Effect of prenatal exposure to polychlorinated biphenyls on incidence of acute respiratory infections in preschool Inuit children. Environmental Health Perspectives, v.114, n.8, p.1301-1305, 2006. Disponível em: <http://dx.doi.org/10.1289/ehp.9084>. Acesso em: 28 nov. 2011. doi: $10.1289 / \mathrm{ehp} .8683$.

EFSA. European Food Safety Authority. Opinion of the scientific panel on contaminants in the food chain on a request from the commission related to the presence of non dioxinlike polychlorinated biphenyls (PCB) in feed and food. European Food Safety Authority Journal, n.284, p.1-137, 2005. Disponível em: <http://www.efsa.europa.eu/en/ efsajournal/pub/284.htm>. Acesso em: 28 nov. 2011. doi: $10.2903 /$ j.efsa.2005.284. 
ERICKSON, M.D.; KALEY II, R.G. Applications of polychlorinated biphenyls. Environmental Science and Pollution Research, v.18, n.2, p.135-151, 2011. Disponível em: <http://www.springerlink.com/content/43r52v1238412618/>. Acesso em: 28 nov. 2011. doi: 10.1007/s11356-010-0392-1.

FERRANTE, M.C. et al. Polychlorinated biphenyls and organochlorine pesticides in European eel (Anguilla Anguilla) from the Garigliano River (Campania region, Italy). Chemosphere, v.78, n.6, p.709-716, 2010. Disponível em: $<\mathrm{http}: / / \mathrm{ww}$ w.sciencedirect.com/science/article/pii/ S0045653509013836>. Acesso em: 28 nov. 2011. doi: 10.1016/j.chemosphere.2009.11.026.

FERRANTE, M.C. et al. Effects of non-dioxin-like polychlorinated biphenyl congeners (PCB 101, PCB 153 and PCB 180) alone or mixed on J774A.1 macrophage cell line: modification of apoptotic pathway. Toxicology Letters, v.202, n.1, p.61-68, 2011. Disponível em: <http:// www.sciencedirect.com/science/article/pii/ S0378427411000257>. Acesso em: 28 nov. 2011. doi: $10.1016 /$ j.toxlet.2011.01.023.

FROMBERG, A. et al. Estimation of dietary intake of PCB and organochlorine pesticides for children and adults. Food Chemistry, v.125, n.4, p.1179-1187, 2011. Disponível em: $<$ http://www.sciencedirect.com/science/article/pii/ S0308814610012720>. Acesso em: 28 nov. 2011. doi: 10.1016/j.foodchem.2010.10.025.

GÓMARA, B. et al. Occurrence of co-planar polybrominated/ chlorinated biphenyls (PXBs), polybrominated diphenyl ethers (PBDEs) and polychlorinated biphenyls (PCBs) in breast milk of women from Spain. Chemosphere, v.83, n.6, p.799-805, 2011. Disponível em: $<\mathrm{http}: / / \mathrm{www} . s c i e n c e d i r e c t . c o m / s c i e n c e /$ article/pii/S004565351100244X>. Acesso em: 28 nov. 2011. doi: $10.1016 /$ j.chemosphere.2011.02.080.

HECK, M.C. et al. Estimation of children exposure to organochlorine compounds through milk in Rio Grande do Sul, Brazil. Food Chemistry, v.102, n.1, p.288-294, 2007. Disponível em: <http://www.sciencedirect.com/science/article/ pii/S0308814606004109>. Acesso em: 28 nov. 2011. doi: 10.1016/j.foodchem.2006.05.019.

HUE, O. et al. Increased plasma levels of toxic pollutants accompanying weight loss induced by hipocaloric diet or by bariatric surgery. Obesity Surgery, v.16, n.9, p.1145-1154, 2006. Disponível em: <http://www.springerlink.com/content/ $60022 \mathrm{~m} 76182 \mathrm{v} 2493 />$. Acesso em: 28 nov. 2011 . doi: $10.1381 / 096089206778392356$.

KIM, M.J. et al. Fate and complex pathogenic effects of dioxins and polychlorinated biphenyls in obese subjects before and after drastic weight loss. Environmental Health Perspectives, v.119, n.3, p.377-383, 2011. Disponível em: <http://dx.doi.org/ 10.1289/ehp.1002848>. Acesso em: 28 nov. 2011. doi: $10.1289 /$ ehp. 1002848

LEVIN, M. et al. Non-coplanar PCB-mediated modulation of human leukocyte phagocytosis: a new mechanism for immunotoxicity. Journal of Toxicology and Environmental Health A, v.68, n.22, p.1977-1993, 2005. Disponível em: $<\mathrm{http}$ ://www.tandfonline.com/doi/abs/10.1080/ 15287390500227126>. Acesso em: 28 nov. 2011. doi: $10.1080 / 15287390500227126$.
LUEBKE, R.W. et al. Suppression of immune function and susceptibility to infections in humans: association of immune function with clinical disease. Journal of Immunotoxicology, v.1, n.1, p.15-24, 2004. Disponível em: <http:// informahealthcare.com/toc/imt/1/1>. Acesso em: 28 nov. 2011. doi: $10.1080 / 15476910490438342$.

MAERVOET, J. et al. A reassessment of the nomenclature of polychlorinated biphenyl (PCB) metabolites. Environmental Health Perspectives, v.112, n.3, p.291-294, 2004. Disponível em: $<$ http://dx.doi.org/10.1289/ehp.6409>. Acesso em: 28 nov. 2011. doi: 10.1289/ehp.6409.

MARTÍ, M. et al. Persistent organic pollutants (PCDD/Fs, dioxin-like PCBs, marker PCBs, and PBDEs) in health supplements on the Spanish market. Chemosphere, v.78, n.10, p.1256-1262, mar. 2010. Disponível em: <http:// www.sciencedirect.com/science/article/pii/ S0045653509014611>. Acesso em: 28 nov. 2011. doi: 10.1016/j.chemosphere.2009.12.038.

MIDIO, A.F.; MARTINS, I.S. Toxicologia de alimentos. São Paulo: Varela, 2000. 295p.

MOHR, S. et al. Determinação de bifenilos policlorados em soro de cordão umbilical através de extração por hidrólise ácida seguida de cromatografia a gás acoplada a um microdetector de captura de elétrons. Química Nova, v.34, n.3, p.444-449, 2011. Disponível em: $<\mathrm{http}: / / \mathrm{www} . \mathrm{scielo} \cdot \mathrm{br} / \mathrm{pdf} / \mathrm{qn} / \mathrm{v} 34 \mathrm{n} 3 /$ 15.pdf>. Acesso em: 28 nov. 2011. doi: 10.1590/S010040422011000300015.

OMAYE, S.T. Food and nutritional toxicology. Boca Ranton: CRC, 2004. 319p.

ONDARZA, P.M. et al. Polybrominated diphenyl ethers and organochlorine compound levels in Brown trout (Salmo trutta) from Andean Patagonia, Argentina. Chemosphere, v.83, n.11, p.1597-1602, 2011. Disponível em: <http:// www.sciencedirect.com/science/article/pii/ S0045653511000075>. Acesso em: 28 nov. 2011. doi:10.1016/ j.chemosphere.2011.01.005.

PENTEADO, J.C.; VAZ, J.M. O legado das bifenilas policloradas (PCBs). Química Nova, v.24, n.3, p.390-398, 2001. Disponível em: <http://www.scielo.br/pdf/qn/v24n3/ a16v24n3.pdf $>$. Acesso em: 28 nov. 2011. doi: 10.1590/ S0100-40422001000300016.

PEREIRA, M.S. Polychlorinated dibenzo-p-dioxins (PCDD), dibenzofurans (PCDF) and polychlorinated biphenyls (PCB): main sources, environmental behaviour and risk to man and biota. Química Nova, v.27, n.6, p.934-943, 2004. Disponível em: <http://www.scielo.br/pdf/qn/v27n6/22284.pdf >. Acesso em: 28 nov. 2011. doi: 10.1590/S0100-40422004000600018.

PRINCE, M.M. et al. Mortality and exposure response among 14.458 electrical capacitor manufacturing workers exposed to polychlorinated biphenys (PCBs). Environmental Health Perspectives, v.114, n.10, p.1508-1514, 2006. Disponível em: <http://dx.doi.org/10.1289/ehp.9175>. Acesso em: 28 nov. 2011. doi: 10.1289/ehp.9175.

QIN, Y.Y. et al. Persistent organic pollutants in food items collected in Hong Kong. Chemosphere, v.82, n.9, p.13291336, 2011. Disponível em: <http://www.sciencedirect.com/ 
science/article/pii/S0045653510013998>. Acesso em: 28 nov. 2011. doi: 10.1016/j.chemosphere.2010.12.009.

RECIO-VEGA, R. et al. Serum levels of polychlorinated biphenyls in Mexican women and breast cancer risk. Journal of Applied Toxicology, v.31, n.3, p.270-278, 2011. Disponível em: <http://onlinelibrary.wiley.com/doi/10.1002/jat.1672/ abstract>. Acesso em: 28 nov. 2011. doi: 10.1002/jat.1672.

ROSS, G. The public health implications of polychlorinated biphenyls (PCBs) in the environment. Ecotoxicology and Environmental Safety, v.59, n.3, p.275-291, 2004. Disponível em: <http://www.sciencedirect.com/science/article/ pii/S0147651304000909>. Acesso em: 28 nov. 2011. doi: 10.1016/j.ecoenv.2004.06.003.

SAFE, S. et al. PCBs: structure-function relationships and mechanism of action. Environmental Health Perspectives, v.60, p.47-56, 1985. Disponível em: <http://dx.doi.org/ 10.1289/ehp.856047>. Acesso em: 28 nov. 2011. doi: 10.1289/ ehp. 856047.

SAFE, S.H. Comparative toxicology and mechanism of action of polychlorinated dibenzo-p-dioxins and dibenzofurans. Annual Review of Pharmacology and Toxicology, v.26, p.371-399, 1986. Disponível em: <http:// www.annualreviews.org/doi/abs/10.1146/ annurev.pa.26.040186.002103>. Acesso em: 28 nov. 2011. doi: 10.1146/annurev.pa.26.040186.002103.

SANTOS, J.S. et al. Assessment of polychlorinated biphenyls (PCBs) in cheese from Rio Grande do Sul, Brazil. Chemosphere, v.65, n.9, p.1544-1550, 2006. Disponível em: $\quad<$ http://www.sciencedirect.com/science/article/pii/ S0045653506004255>. Acesso em: 28 nov. 2011. doi: 10.1016/j.chemosphere.2006.04.003.

SELGRADE, M.K. Immunotoxicity: the risk is real. Toxicological Sciences, v.100, n.2, p.328-332, 2007. Disponível em: <http://toxsci.oxfordjournals.org/content/100/ 2/328.full>. Acesso em: 28 nov. 2011. doi: 10.1093/toxsci/ $\mathrm{kfm} 244$.

SILVA, C.E.A. et al. Toxicologia das bifenilas policloradas. Oecologia Brasiliensis, v.11, n.2, p.179-187, 2007.

STORELLI, M.M.; PERRONE, V.G. Detection and quantitative analysis of organochlorine compounds (PCBs and DDTs) in deep sea fish liver from Mediterranean Sea. Environmental Science and Pollution Research, v.17, n.4, p.968-976, 2010. Disponível em: <http://www.springerlink.com/content/ x384658156724021/>. Acesso em: 28 nov. 2011. doi: 10.1007/ s11356-010-0300-8.

TOKADA, T. et al. Relationship between the concentrations of polychlorinated dibenzo-p-dioxins, polychlorinated dibenzofurans, and polychlorinated biphenyls in maternal blood and those in breast milk. Chemosphere, v.78, n.2, p.185192, 2010. Disponível em: <http://www.sciencedirect.com/ science/article/pii/S0045653509011394>. Acesso em: 28 nov. 2011. doi: 10.1016/j.chemosphere.2009.09.047.

TURRIO-BALDASSARI, L. et al. PCB, PCDD and PCDF contamination of food of animal origin as the effect of soil pollution and the cause of human exposure in Brescia. Chemosphere, v.76, n.2, p.278-285, 2009. Disponível em: $<$ http://www.sciencedirect.com/science/article/pii/ S0045653509002896>. Acesso em: 28 nov. 2011. doi: 10.1016/j.chemosphere.2009.03.002.

VASSILIADOU, I. et al. Dioxin contamination after an accidental fire in the municipal landfill of Tagarades, Thessaloniki, Greece. Chemosphere, v.74, n.7, p.879-884, 2009. Disponível em: <http://www.sciencedirect.com/science/ article/pii/S0045653508014124>. Acesso em: 28 nov. 2011. doi: 10.1016/j.chemosphere.2008.11.016.

VOORSPOELS, S. et al. Dietary PCB intake in Belgium. Environmental Toxicology and Pharmacology, v.25, n.2, p.179-182, 2008. Disponível em: <http:// www.sciencedirect.com/science/article/pii/ S1382668907001639>. Acesso em: 28 nov. 2011. doi: 10.1016/j.etap.2007.10.013.

WEBER, R. et al. Dioxin - contemporary and future challengers of historical legacies. Environmental Science and Pollution Research, v.15, n.2, p.96-100, 2008. Disponível em: <http:/ /www.springerlink.com/content/9032774680846044/>. Acesso em: 28 nov. 2011. doi: 10.1065/espr2008.01.473.

WHO. World Health Organization. Polychlorinated biphenyls and terphenyls. Environmental Health Criteria: 140. Geneva: World Health Organization, 1993. 682p.

WHO. World Health Organization. Polychlorinated biphenyls: human health aspects. Disponível em: <http:/ /www.inchem.org/documents/cicads/cicads/cicad55.htm>. Online. Acesso em: 28 nov. 2011.

XING, G.H. et al. Environmental impact and human exposure to PCBs in Guiyu, an electronic waste recycling site in China. Environment International, v.35, n.1, p.76-82, 2009. Disponível em: <http://www.sciencedirect.com/science/article/ pii/S0160412008001505>. Acesso em: 28 nov. 2011. doi: 10.1016/j.envint.2008.07.025.

YAGÜE, C. et al. Determination of pesticides and PCBs in virgin olive oil by multicolumn solid-phase extraction cleanup followed by GC-NPD/ECD and confirmation by ion-trap GCMS. Journal of Agricultural and Food Chemistry, v.53, n.13, p.5105-5109, 2005. Disponível em: <http://pubs.acs.org/ doi/abs/10.1021/jf0502471>. Acesso em: 28 nov. 2011. doi: $10.1021 / \mathrm{jf050247I.}$ 